\title{
Differential View on the Bile Acid Stress Response of Clostridioides difficile
}

\author{
Susanne Sievers ${ }^{1 *}$, Nicole G. Metzendorf ${ }^{1}$, Silvia Dittmann ${ }^{1}$, Daniel Troitzsch ${ }^{1}$, \\ Viola Gast ${ }^{1}$, Sophie Marlen Tröger ${ }^{1}$, Christian Wolff', Daniela Zühlke', \\ Claudia Hirschfeld ${ }^{2}$, Rabea Schlüter ${ }^{3}$ and Katharina Riedel ${ }^{1}$
}

1 Institute of Microbiology, Center for Functional Genomics of Microbes, University of Greifswald, Greifswald, Germany, ${ }^{2}$ Department of Microbial Proteomics, Institute of Microbiology, Center for Functional Genomics of Microbes, University of Greifswald, Greifswald, Germany, ${ }^{3}$ Imaging Center of the Department of Biology, University of Greifswald, Greifswald, Germany

\section{OPEN ACCESS}

Edited by: George Grant University of Aberdeen, United Kingdom

Reviewed by:

Elizabeth Karr

The University of Oklahoma,

United States

Wolfgang Buckel,

University of Marburg, Germany

${ }^{*}$ Correspondence:

Susanne Sievers

susanne.sievers@uni-greifswald.de

Specialty section:

This article was submitted to Infectious Diseases,

a section of the journal

Frontiers in Microbiology

Received: 26 September 2018

Accepted: 31 January 2019

Published: 18 February 2019

Citation:

Sievers S, Metzendorf NG,

Dittmann S, Troitzsch D, Gast V,

Tröger SM, Wolff C, Zühlke $D$,

Hirschfeld C, Schlüter R and Riedel K

(2019) Differential View on the Bile

Acid Stress Response

of Clostridioides difficile.

Front. Microbiol. 10:258.

doi: 10.3389/fmicb.2019.00258
Clostridioides difficile is an intestinal human pathogen that uses the opportunity of a depleted microbiota to cause an infection. It is known, that the composition of the intestinal bile acid cocktail has a great impact on the susceptibility toward a $C$. difficile infection. However, the specific response of growing $C$. difficile cells to diverse bile acids on the molecular level has not been described yet. In this study, we recorded proteome signatures of shock and long-term (LT) stress with the four main bile acids cholic acid (CA), chenodeoxycholic acid (CDCA), deoxycholic acid (DCA), and lithocholic acid (LCA). A general overlapping response to all tested bile acids could be determined particularly in shock experiments which appears plausible in the light of their common steroid structure. However, during LT stress several proteins showed an altered abundance in the presence of only a single or a few of the bile acids indicating the existence of specific adaptation mechanisms. Our results point at a differential induction of the groEL and dnaKJgrpE chaperone systems, both belonging to the class I heat shock genes. Additionally, central metabolic pathways involving butyrate fermentation and the reductive Stickland fermentation of leucine were effected, although CA caused a proteome signature different from the other three bile acids. Furthermore, quantitative proteomics revealed a loss of flagellar proteins in LT stress with LCA. The absence of flagella could be substantiated by electron microscopy which also indicated less flagellated cells in the presence of DCA and CDCA and no influence on flagella formation by $C A$. Our data break down the bile acid stress response of $C$. difficile into a general and a specific adaptation. The latter cannot simply be divided into a response to primary and secondary bile acids, but rather reflects a complex and variable adaptation process enabling $C$. difficile to survive and to cause an infection in the intestinal tract.

Keywords: Clostridioides difficile, proteomics, bile acids, motility, flagella, stress response, chaperones

\section{INTRODUCTION}

The anaerobic bacterium Clostridioides difficile represents one of the most serious nosocomial pathogens and is the main cause of antibiotics-associated diarrhea (Thomas et al., 2003). Two main toxins (Toxins A and B) provoke a disruption of the intestinal epithelium and a strong inflammatory host response leading to symptoms from mild diarrhea to more serious and often 
life-threatening conditions such as pseudomembranous colitis, toxic megacolon and eventually an intestinal perforation (Bartlett, 2006; Rupnik et al., 2009). The expression level of toxins in C. difficile was shown to be not only strain dependent, but also tightly connected to the growth state and basic physiology of the bacterium (Karlsson et al., 2008; Martin-Verstraete et al., 2016).

As an intestinal pathogen $C$. difficile has to deal with high concentrations of different bile acids, amphiphilic substances with a steroid nucleus (Figure 1). Bile acids are produced by the liver in order to facilitate absorption and digestion of dietary lipids. Due to their soap-like character, bile acids act as natural antimicrobials and only organisms adapted to the challenge will survive in the intestines (Begley et al., 2005). The two main bile acids produced in the human liver are cholic acid (CA) and chenodeoxycholic acid (CDCA) mostly conjugated to taurine or glycine. Species of the intestinal microbiota are capable of deconjugating the primary bile acids, and by dehydroxylation at C7 they can convert CA and CDCA to secondary bile acids resulting in deoxycholic acid (DCA) and lithocholic acid (LCA), respectively (Figure 1). Thus, the microbiota largely contributes to the shaping of the intestinal bile acid composition (Long et al., 2017).

Thirty-five years ago, it was described that bile acid preparations can stimulate the germination of $C$. difficile spores (Wilson, 1983). However, it took another 25 years until Sorg and Sonenshein (2008) elucidated CA as the active component of bile to instigate germination. Not much later they discovered an inhibitory effect of CDCA and analogs of it on C. difficile spore germination (Sorg and Sonenshein, 2009). In 2013, Francis et al. identified the receptor $\mathrm{CspC}$ on the $C$. difficile spore that directly interacts with CA to initiate germination (Francis et al., 2013). Hitherto, no further direct protein-bile acid interactions in $C$. difficile have been described. However, interesting findings on a negative effect of bile acids on the action of $C$. difficile toxins point at a possible direct interaction of the two (Brandes et al., 2012; Darkoh et al., 2013).

Besides the positive effect of CA on spore germination, an inhibitory effect of secondary bile acids not only on germination

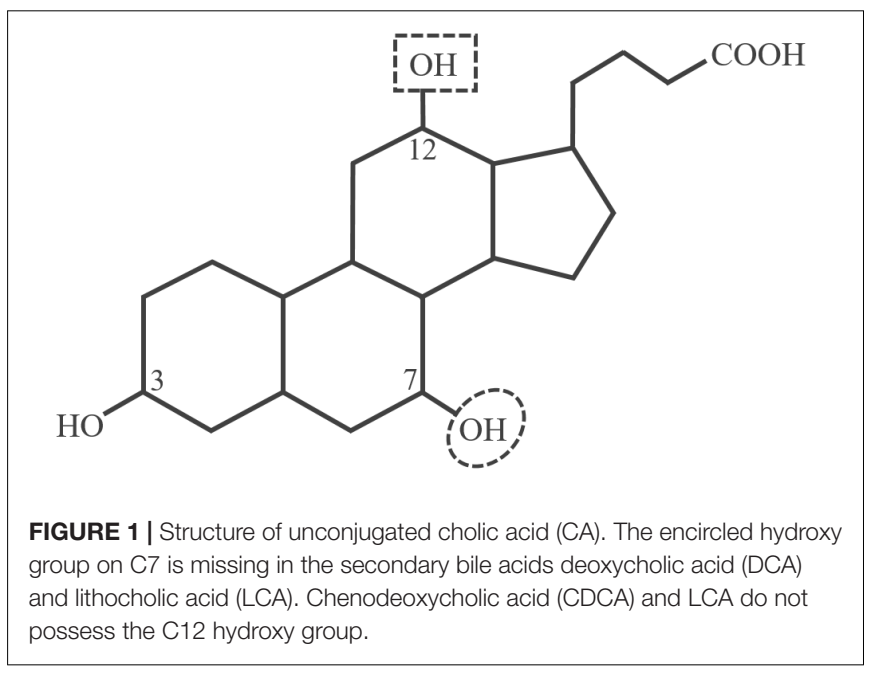

but also on growth and virulence of $C$. difficile has been frequently described (Lewis et al., 2016; Winston and Theriot, 2016; Thanissery et al., 2017). In light of this, the association of a depleted microbiota, which involves an increased ratio of primary to secondary bile acids, and the increased susceptibility to a $C$. difficile infection becomes evident. Very recently, Lewis et al. (2017) could even show that $C$. difficile strains with a higher tolerance for secondary bile acids exhibit a greater disease severity in mice and humans.

During the last years, research focused on unraveling the enzymatic and metabolic crosstalk between species of the microbiota and C. difficile which already led to the identification of some key players including their metabolic abilities in this complex network (Theriot et al., 2014; Buffie et al., 2015; Greathouse et al., 2015; Ridlon et al., 2016; Theriot et al., 2016; Yoon et al., 2017). The gained knowledge is a starting point for the development of personalized and disease-specific strategies to manipulate a patient's intestinal microbiota in the most favorable way (Forster and Lawley, 2015) and to provide an alternative treatment option to the successful but still not fully understood fecal microbiota transplantation (Weingarden et al., 2014; Seekatz et al., 2018).

The microbiota must be studied as a complex system, and certainly the single bricks of this entity influence and depend on each other. Still, in order to fully understand the processes and dependencies within the highly complex intestinal microbial community, single species including their gene expression, physiological abilities and responses upon stimuli have to be investigated (Theriot, 2018).

In this study, we aimed at the characterization of $C$. difficile's stress response to the four main human bile acids. Although, a phenotypic description of growth differences upon challenge with different bile acids has been published (Lewis et al., 2016; Thanissery et al., 2017), no information on the adaptation of gene expression is available until now. In this study, a comprehensive proteomics approach to record stress signatures of the unconjugated bile acids CA, CDCA, DCA and LCA in shock experiments as well as during LT stress conditions has been employed revealing a general stress response to all four bile acids, but also specific responses to only one or a few of the different bile acids.

The specific bile acid compositions of patients may affect germination and growth of $C$. difficile differently. Knowledge on the specific responses of $C$. difficile to different bile acids and on bile acid abundance in a patient could allow speculation on the susceptibility to $C$. difficile and the course of an infection.

\section{MATERIALS AND METHODS}

\section{Strains, Media and Growth Conditions}

For bile acids shock experiments $C$. difficile 630 $\Delta$ erm (Hussain et al., 2005) was inoculated to an $A_{600}$ of 0.05 and grown anaerobically at $37^{\circ} \mathrm{C}$ in brain heart infusion medium (BHI, Oxoid). At an $\mathrm{A}_{600}$ of 0.4 the cultures were shocked with sub-lethal concentrations of the sodium salts of cholic acid [6 mM CA (Sigma-Aldrich)], chenodeoxycholic acid [0.6 mM 
CDCA, (Sigma-Aldrich)], deoxycholic acid [0.6 mM DCA, (Sigma-Aldrich)], and lithocholic acid [0.08 mM LCA, (Steraloids Inc., RI, United States)] finally in solution, or left untreated. After $90 \mathrm{~min}$, cells were harvested anaerobically on ice, pelleted by centrifugation and washed twice in ice-cold, oxygen-free PBS ( $\mathrm{pH}$ 7.2) buffer. In long-term (LT)-stress experiments $C$. difficile $630 \Delta$ erm was inoculated to an $\mathrm{A}_{600}$ of 0.01 in BHI and grown in the presence of varying concentrations of each of the four bile salts over a period of $18 \mathrm{~h}$. Bile acid stressed C. difficile cells and unstressed cells were sampled in exponential growth phase $(15 \mathrm{mM} \mathrm{CA}$ at $A_{600}=0.3,0.8 \mathrm{mM}$ CDCA at $A_{600}=0.3,0.8 \mathrm{mM} \mathrm{DCA}$ at $A_{600}=0.3,0.08 \mathrm{mM} \mathrm{LCA}$ at $A_{600}=0.8$ and untreated at $\left.A_{600}=0.8\right)$, pelleted and washed as previously described (Sievers et al., 2018). Although sodium salts of the bile acids were used in this study, they are referred to as bile acids throughout the manuscript.

\section{Protein Extraction and MS Sample Preparation}

Cell lysis and acetone precipitation of proteins was carried out as previously described (Otto et al., 2016). In brief, cell pellets were resuspended in $50 \mathrm{mM}$ TrisHCl, $\mathrm{pH} 8$ containing $8 \mathrm{M}$ urea, $3 \mathrm{M}$ thiourea, $10 \mathrm{mM}$ EDTA, 4\% CHAPS and $20 \mathrm{mM}$ Tris (2-carboxyethyl)phosphine (TCEP). Cells were lysed by 6 cycles of $1 \mathrm{~min}$ intervals of ultrasonication (Sonotrode MS73, Bandelin, Germany) with intermittent cooling. Cell debris was removed by centrifugation, supernatants mixed with water 1:2 and proteins precipitated overnight at $-20^{\circ} \mathrm{C}$ by adding 4 volumes of acetone. Protein pellets were resuspended in $50 \mathrm{mM}$ TEAB (Triethylammonium bicarbonate) containing $0.1 \%(\mathrm{w} / \mathrm{v})$ RapiGest SF (Waters, United States) and the protein concentration determined using Roti Nanoquant (Carl Roth, Germany). $500 \mu \mathrm{g}$ of protein were reduced in $100 \mu \mathrm{l} 50 \mathrm{mM}$ TEAB, 0.1\% RapiGest, $5 \mathrm{mM}$ TCEP for $45 \mathrm{~min}$ at $60^{\circ} \mathrm{C}$. Afterward free thiols were alkylated for $15 \mathrm{~min}$ in the dark with $10 \mathrm{mM}$ iodoacetamide. For an in-solution digestion of proteins, trypsin was added in a ratio of $1: 100$ and digests incubated in a thermo mixer for $5 \mathrm{~h}$ at $37^{\circ} \mathrm{C}$ and $900 \mathrm{rpm}$. Digestion was stopped and RapiGest removed as recommended by the manufacturer. Sample Peptides were cleaned via StageTipping as described elsewhere (Rappsilber et al., 2007). Purified peptides were dissolved in $100 \mu \mathrm{l}$ of $2 \%(\mathrm{v} / \mathrm{v})$ acetonitrile, $0.1 \%(\mathrm{v} / \mathrm{v})$ acetic acid containing $50 \mathrm{fmol} / \mu \mathrm{l}$ spiked-in yeast alcohol dehydrogenase (Waters).

\section{LC-MSE Analysis}

Peptides were analyzed using a nanoACQUITY ${ }^{\mathrm{TM}}$ UPLC $^{\mathrm{TM}}$ system (Waters) coupled to a Synapt G2 mass spectrometer (Waters). Details on liquid chromatography and $\mathrm{IMS}^{\mathrm{E}}\left(\mathrm{MS}^{\mathrm{E}}\right.$ with ion mobility separation) methods are described elsewhere (Muntel et al., 2014; Zuhlke et al., 2016). LC-IMS ${ }^{\mathrm{E}}$ data were processed using PLGS v3.0.1. Processing parameters were applied according to Schmidt et al. (2017).
$\mathrm{MS}^{\mathrm{E}}$ raw data were searched against the randomized C. difficile $630 \Delta$ erm database published by Dannheim et al. (2017) containing 3781 protein entries of C. difficile plus laboratory contaminants and the yeast $\mathrm{ADH} 1$ sequence. For a positive protein identification the following criteria had to be met: 1 fragment ion matched per peptide, 5 fragment ions matched per protein, 1 peptide matched per protein; 2 missed cleavages allowed, primary digest reagent: trypsin, fixed modification: carbamidomethylation C (+57.0215), variable modifications: deamidation $\mathrm{N}$ and $\mathrm{Q}$, oxidation $\mathrm{M}$, pyrrolidonecarboxylacid N-TERM. The protein false discovery rate (FDR) was set to $5 \%$ and only identifications based on at least two peptides were considered in the final analysis. Three biological replicates, each run in three technical replicates were conducted for shock experiments. LT stress experiments were performed in 4 biological replicates with three technical replicates of each.

\section{Protein Quantification, Data Evaluation and Visualization}

Data on protein quantities were corrected for detector saturation effects by implementing a correction factor as recently described (Zuhlke et al., 2016). Datasets were normalized on the basis of the spiked-in yeast alcohol dehydrogenase. The mass spectrometry proteomic data have been deposited in the ProteomeXchange Consortium via the PRIDE partner repository (Vizcaino et al., 2016). Results of the shock experiments can be found with dataset identifier PXD010514. LT stress data are linked with identifier PXD010525. The Perseus software platform (Tyanova et al., 2016) was used for the further analysis of proteomic datasets as recently described (Neumann-Schaal et al., 2018). Data were filtered (shock experiments: 3 out of 3 replicates and LT stress experiments: 4 out of 4 replicates within one group of valid values), $\log 2$ transformed and normalized by division by the means to generate volcano plots as well as to carry out ANOVA testing (permutation-based, FDR 0.01 for shock and 0.001 for LT stress experiments) to create heatmaps ( $z$-score normalization, distance euclidean). For principal component analysis (PCA) missing data was imputed (settings: width 0.3, down shift 1.8, for PCA: cut off Benjamini Hochberg FDR 0.05). Interactive 3D plots were generated using the Plotly package in R v. 3.4 .3 (30 November, 2017), (Sievert et al., 2016). Voronoi treemaps were built using the Paver software (DECODON $\mathrm{GmbH}$ ) as previously described (Otto et al., 2016).

\section{Motility Assay}

The influence of bile acids on the swimming motility of $C$. difficile was tested on $10 \mathrm{~cm}$ diameter soft agar plates (BHI with $0.175 \%$ [w/vol] agar). A sigH mutant was used as a non-motile control (Saujet et al., 2011). Plates were inoculated with single colonies that were pierced onto the centers of the plates with increasing concentrations of bile acids. Plates were incubated anaerobically at $37^{\circ} \mathrm{C}$. Diameters of halos due to bacterial migration were measured $48 \mathrm{~h}$ after inoculation. 


\section{Electron Microscopy and Flagella Quantification}

Bile acid conditions and time points of harvest for negative staining were chosen as in LT stress experiments. C. difficile was pelleted by centrifugation $\left(4,000 \times \mathrm{g}, 4^{\circ} \mathrm{C}, 10 \mathrm{~min}\right)$. Cells were washed once with sterile-filtered $(0.2 \mu \mathrm{m}) 1 \times$ PBS and fixed (2.5\% glutaraldehyde, $2 \%$ paraformaldehyde in PBS) for $1 \mathrm{~h}$ at RT followed by at least $12 \mathrm{~h}$ at $4^{\circ} \mathrm{C}$. Samples were centrifuged and washed three times with sterile-filtered $1 \times$ PBS. Pellets were resuspended in sterile-filtered $1 \times$ PBS. The flotation method was used for the negative staining procedure. Fixed cells were allowed to adsorb onto a glow-discharged Pioloform carbon-coated 400mesh grid for $10 \mathrm{~min}$. The grid was then transferred onto two droplets of deionized water, and finally onto a drop of $0.5 \%$ aqueous uranyl acetate for $10 \mathrm{~s}$. After blotting with filter paper and air-drying, the samples were examined with a transmission electron microscope LEO 906 (Carl Zeiss Microscopy GmbH) at an acceleration voltage of $80 \mathrm{kV}$. Sharpness and contrast of micrographs were adjusted by using Adobe Photoshop CS6. Transmission electron micrographs at a 1293 -fold magnification were analyzed with Fiji (Schindelin et al., 2012) to determine the length and width of the cells as well as the total length of all visible flagella. For the investigation of the area covered with flagella, a grate with squares $\left(1.6 \mu \mathrm{m}^{2}\right)$ was applied to all images. Squares with flagella were counted as positive. All data were further analyzed using Microsoft Excel. Statistical significance was assessed by an unpaired Student's $t$-Test.

\section{RESULTS}

\section{Growth-Inhibiting Effect of Bile Acids}

To determine inhibitory concentrations of bile acids in C. difficile two different types of stress experiments were conducted. First, sub-lethal concentrations of the four different bile acids were determined in shock experiments, i e., exponentially growing cells were shocked with concentrations that prevented further growth of $C$. difficile, but did not cause lysis. Sub-lethal concentrations of CA, DCA and CDCA were defined as $6,0.6$ and $0.6 \mathrm{mM}$, respectively. Thus, CA could be added in an amount 10 times higher compared to DCA and CDCA until causing the same effect on growth. LCA was used at a maximum concentration of $0.08 \mathrm{mM}$. This caused only a slight decrement of growth, but higher concentrations of LCA resulted in formation of micelles and thereby impaired measurements of the optical density.

In a second experimental setup C. difficile was subjected to long-term bile acid stress. To this end, the bacterium was inoculated with a low starting $A_{600}$ of 0.01 into medium already containing the bile acids. Different concentrations of the single bile acids were tested and growth was measured over a period of $20 \mathrm{~h}$ until cultures reached stationary phase to identify concentrations of each bile acid that cause a strong and comparably inhibitory effect allowing the cultures to grow up to an $\mathrm{A}_{600}$ of about 1 (Supplementary Figure S1). LCA was again used at the maximum possible concentration of $0.08 \mathrm{mM}$ which had only a minor effect on the growth of $C$. difficile compared to control conditions. The other three bile acids were applied in concentrations considerably higher than the sub-lethal ones used in shock experiments pointing at an ability of $C$. difficile to adapt to the presence of bile acids. Also in LT stress experiments, much higher concentrations of CA were tolerated compared to DCA and CDCA. However, C. difficile cells grown in presence of CA showed a strikingly longer lag-phase compared to the DCA and CDCA-treated cultures (Supplementary Figure S1).

\section{Differential Response to Bile Acids}

\section{Shock Response}

For comparative proteome analyses, C. difficile cultures shocked with sub-lethal concentrations of bile acids determined above were harvested together with an untreated control culture after 90 min of shock onset. The $C$. difficile culture shocked with $0.08 \mathrm{mM}$ LCA kept growing almost to the same extent as the control. Still, in a principal component analysis (PCA) comprising all three replicates of all five conditions, the control samples clearly separate from LCA stress and all other stresses and CA and DCA cluster together apart from CDCA and LCA (Figure 2A). Hence, a primary bile acid clusters closer with its corresponding secondary bile acid than with the other primary bile acid.

\section{LT Stress Response}

For comparative proteome analyses, C. difficile cells were constantly grown in absence (controls) and presence of bile acids, and samples for comprehensive proteome analyses were taken in the late-exponential growth phase in four replicates for each condition (15 mM CA, $0.8 \mathrm{mM}$ CDCA, $0.8 \mathrm{mM}$ DCA, $0.08 \mathrm{mM}$ LCA). As observed in shock experiments, the expression profiles of the control cultures clearly differ from those of bile acid-stressed cultures. However, these LT stress experiments also clearly delineate the changes in the expression profiles of the different bile acids, whereas biological replicates correspond very well (Figure 2B). Since all cells were harvested during late-exponential growth, the observed differences in the protein profiles correspond to specific stress responses caused by each of the tested bile acids.

\section{Bile Acids Induce a General and Specific Response \\ Proteome Shock Signatures}

Proteins that were quantified in 3 out of 3 replicates were subjected to an ANOVA analysis to identify differentially expressed proteins comparing bile acid conditions with control but also bile acid conditions among each other (Supplementary Tables S1, S2). 76 proteins met the criterion of a permutation based FDR of 0.01 and thus appeared to be differentially abundant between the tested conditions (Figure 3A). Approximately one third of these proteins represent a general bile acid stress response, i.e., their abundance proved to be higher in presence of each of the tested bile acids compared to control conditions. Amongst these are chaperones DnaK/DnaJ/GrpE, cell wall binding proteins (Cwp2, Cwp66, Cwp22) and proteins involved in cell division (FtsZ, FtsH2). 


\section{A}

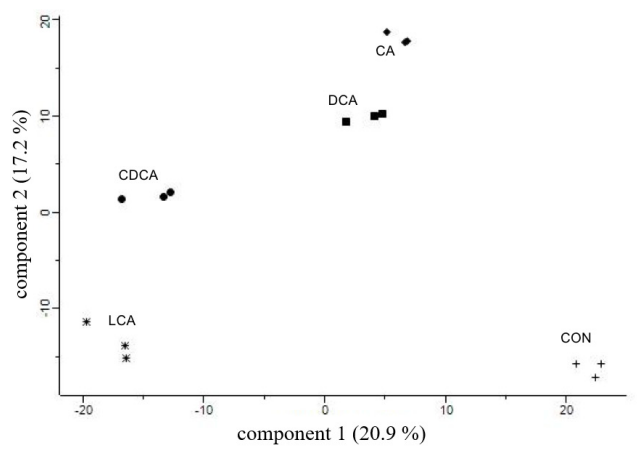

B

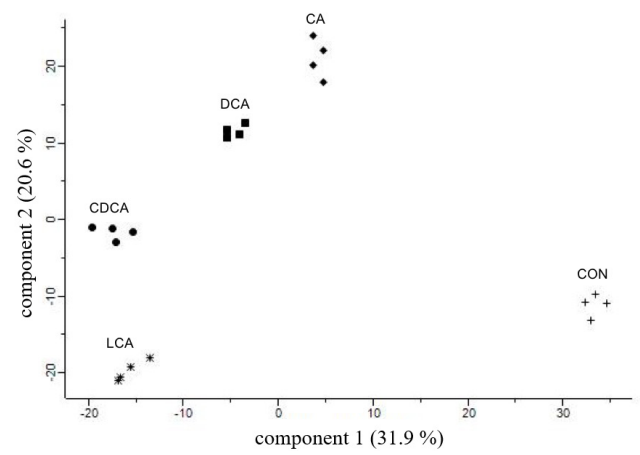

FIGURE 2 | Global proteomic datasets of shock experiments (A) and LT stress (B) depicted in PCAs. Bile acids are abbreviated as follows: CA, cholic acid; DCA, deoxycholic acid; CDCA, chenodeoxycholic acid; LCA, lithocholic acid; CON, unstressed culture.

A

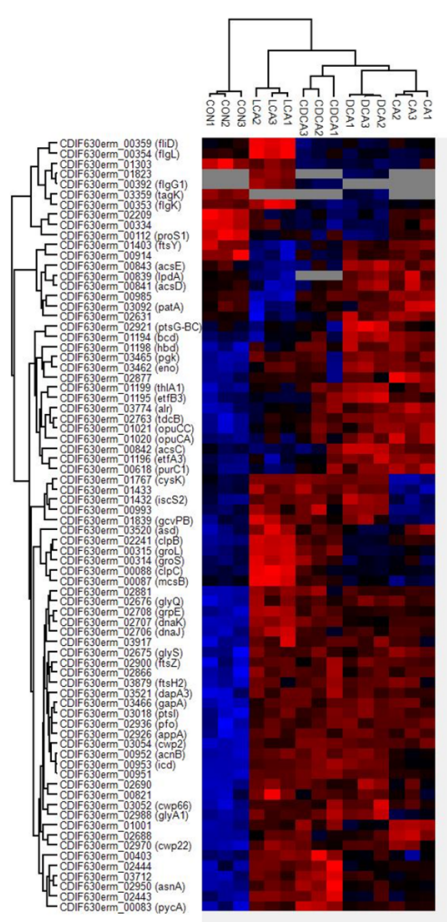

B

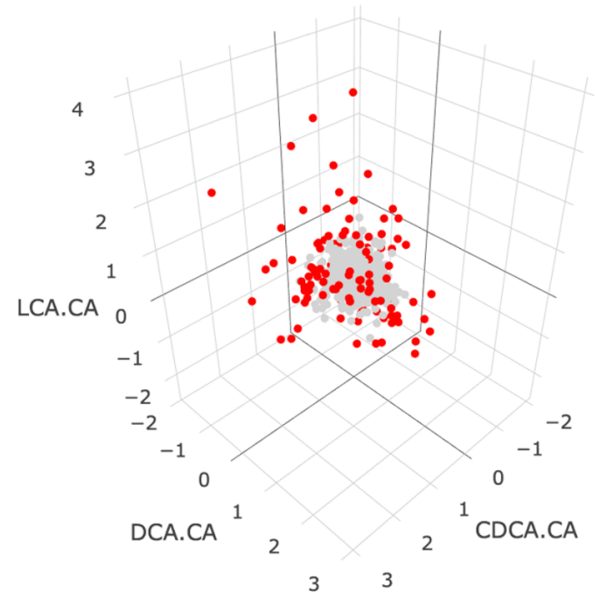

FIGURE 3 | Heatmap comprising all three replicates of control and the four bile acid samples (A). Proteins which were not quantified in all three replicates of a specific condition were not considered indicated by gray coloring. Red and blue colors indicate high and low protein abundance, respectively. A 3-D plot of ratios of protein abundance $[\Delta \log 2(\mathrm{LCA}-\mathrm{CA}), \Delta \log 2(\mathrm{CDCA}-\mathrm{CA}), \Delta \log 2(\mathrm{DCA}-\mathrm{CA})]$ for proteins which could be quantified in all four different bile shock conditions is shown (B). Protein dots for which the log2 ratio is higher than 1 or below -1 are shown in red (FC $\geq 2)$. A rotatable 3D plot is provided supplementary

(Supplementary Figure S3).

Notably, the abundance of several proteins differed significantly depending on the kind of bile acid used in the shock experiment indicating bile acid-specific responses. To better identify these differentially expressed proteins, protein amounts of each condition were compared to every other condition and visualized in volcano plots and Voronoi treemaps (Supplementary Figure S2). Furthermore, ratios of LCA vs. CA, DCA vs. CA and CDCA vs. CA were calculated and visualized in a $3 \mathrm{D}$-plot
(Figure 3B). Data points close to the origin represent proteins similarly expressed amongst different bile acids, but the longer the distance between data point and origin, the more distinctive is the response to a specific bile acid. An animated and rotatable version of Figure 3B is provided supplementary (Supplementary Figure S3). Surprisingly, and in contrast to the DnaK/DnaJ/GrpE system, the chaperonins GroL and GroS as well as chaperone $\mathrm{ClpB}$, the ATPase $\mathrm{ClpC}$ and the associated arginine kinase McsB 
are not generally induced by bile acids. These proteins highly increase in amount after shock with LCA and extenuated after CDCA shock, but were not affected by DCA or CA. Moreover, LCA, DCA and CDCA, but not CA seem to interfere with the synthesis of iron sulfur clusters, since protein CysK, which catalyzes the formation of cysteine from $\mathrm{O}$-acetyl-serine and $\mathrm{H}_{2} \mathrm{~S}$, and proteins IscS2, a cysteine desulfurase, and Fe-S cluster assembly protein CDIF630erm_01433 are all higher abundant compared to control conditions. In summary, we identified numerous proteins, expression of which is similarly altered in response to all tested bile acids, but also a significant number of proteins, whose expression seems to be specifically affected by individual bile acids.

\section{Proteome LT Stress Signatures}

For the evaluation of the LT bile acid stress experiment, proteins quantified in 4 out of 4 replicates were chosen for an ANOVA analysis (Supplementary Tables S3, S4). Applying a very stringent and permutation-based FDR of 0.001 still yielded 222 proteins differing in abundance when cells were grown in the absence and presence of different bile acids (Figure 4A).

In contrast to the shock experiments, there was no extensive general bile acids stress response with similar changes in protein abundance across all four LT stress conditions. A central metabolic pathway in $C$. difficile that was affected during LT challenge is the conversion of pyruvate to fermentation products as ethanol, butanol or butanoate (Hofmann et al., 2018). Enzymes of this pathway were quantitatively affected by all tested bile acids, however, not always uniformly or to the same extent (Figure 5A). Apparently, all bile acids provoke a decrease of alcohol dehydrogenases AdhE1 and AdhE2 and thereby hamper the conversion of acetyl-CoA to ethanol or butanol. Enzymes of upstream reactions catalyzing the conversion of acetyl-CoA to butanoyl-CoA are mostly up-regulated by CA and DCA, but enzymes involved in the processing of butanoylCoA to butanoate are up-regulated only in the presence of CDCA, DCA and LCA, but not CA compared to control conditions. Numerous other proteins are more abundant when cells were subjected to continuous stress of the different bile acids with exception of CA (Figure 4A), e.g., VorAA*BC1 involved in the oxidative Stickland reaction of branched chain amino acids as well as proteins belonging to the Gro and Dna chaperone machineries, which contrasts with the results of the shock experiment. Moreover, almost all enzymes of the reductive Stickland fermentation of leucine to isocaproate, representing another key metabolic pathway in $C$. difficile (Hofmann et al., 2018), were found to be induced by all bile acids except CA (Figure 5B).

Almost one third of the 222 differentially expressed proteins follow the pattern of a significant downregulation in LCA and CDCA stressed cells vs. DCA, CA and control conditions. Representative proteins of this cluster are the subunits A2, D2, $\mathrm{H}$, and F2 of the ATP-Synthase complex. Also, several subunits building the proline reductase complex (PrdBAC) are more abundant at CA and DCA compared to LCA and CDCA. However, the major fraction of gene products with such specific regulation pattern is represented by cell wall-bound proteins
(Cwp2/6/18/19/66/84) and proteins known to be involved in the formation of the cell wall (MurA, MreB1, and B2).

As for shock datasets, data of the long-term stress experiments were also compared pairwise in volcano plots and Voronoi treemaps (Supplementary Figure S4) and the ratios of LCA vs. CA, DCA vs. CA and CDCA vs. CA visualized (Figure 4B). This 3D-plot, whose perspective can be conveniently rotated (Supplementary Figure S3), emphasizes the high number of proteins that are subject to a specific regulation during LT bile acid challenge.

\section{Changes in Morphology}

The proteome analysis of LT-stressed cells revealed a dramatic decrease of the major structural flagellum protein FliC in the presence of CDCA and LCA compared to control conditions. Also DCA stress resulted in down-regulation of FliC, but not as dramatically. Interestingly, CA did not provoke any altered fliC expression. Another 22 proteins of the flagellar filament have been identified in this proteome analysis (from gene loci CDIF630erm_00349 down to CDIF630erm_00395), but none of them could be identified in all bile acids stress conditions. Strikingly, 15 of these proteins were identified in all four replicates of control and CA conditions, but not in a single replicate of the other three bile acid stress conditions (Supplementary Table S3). Thus, the expression of these proteins appears to be entirely switched off in the presence of selected bile acids. This observation prompted us to test for the presence of flagella in cells grown in presence of the different bile acids by electron microscopy (Figure 6A). C. difficile is extensively flagellated under control conditions. Notably, the electron micrographs of bile acid-stressed cells correlate very well with protein quantification results. Cells challenged with CA are just as flagellated as unstressed cells, whereas fewer filaments have been observed on DCA- and CDCA- stressed C. difficile, and LCA basically provoked a complete loss of flagellar filaments. To quantify the presence of flagella, the length and the area covered by them were measured in more than 50 cells / condition in electron micrographs (Figure 6B). Besides the change in the number of flagella, electron micrographs also revealed an alteration in cell morphology. Whereas cells at control conditions are $4 \mu \mathrm{m}$ long on average, DCA-, CA- and especially CDCAstressed cells were found to be significantly longer with up to $7 \mu \mathrm{m}$ (Figure 6C).

\section{Decrement of Motility}

The bile acid-specific loss of flagella should lead to a reduced motility of $C$. difficile. Therefore, the motility of the bacterium with increasing concentrations of each bile acid was tested on soft agar plates. An immotile sigH mutant of $C$. difficile was used as a negative control. The maximum concentration of bile acids in the motility assays was chosen up to the growth inhibiting concentrations used in LT-stress experiments (15 mM CA, $0.8 \mathrm{mM}$ CDCA, $0.8 \mathrm{mM}$ DCA, $0.08 \mathrm{mM}$ LCA). Figures 7A-D depict the decreasing motility of $C$. difficile $630 \Delta$ erm, which is per se not very motile, with increasing concentrations of CA, DCA, CDCA, and LCA, respectively. However, a decreasing growth zone in response to the presence 


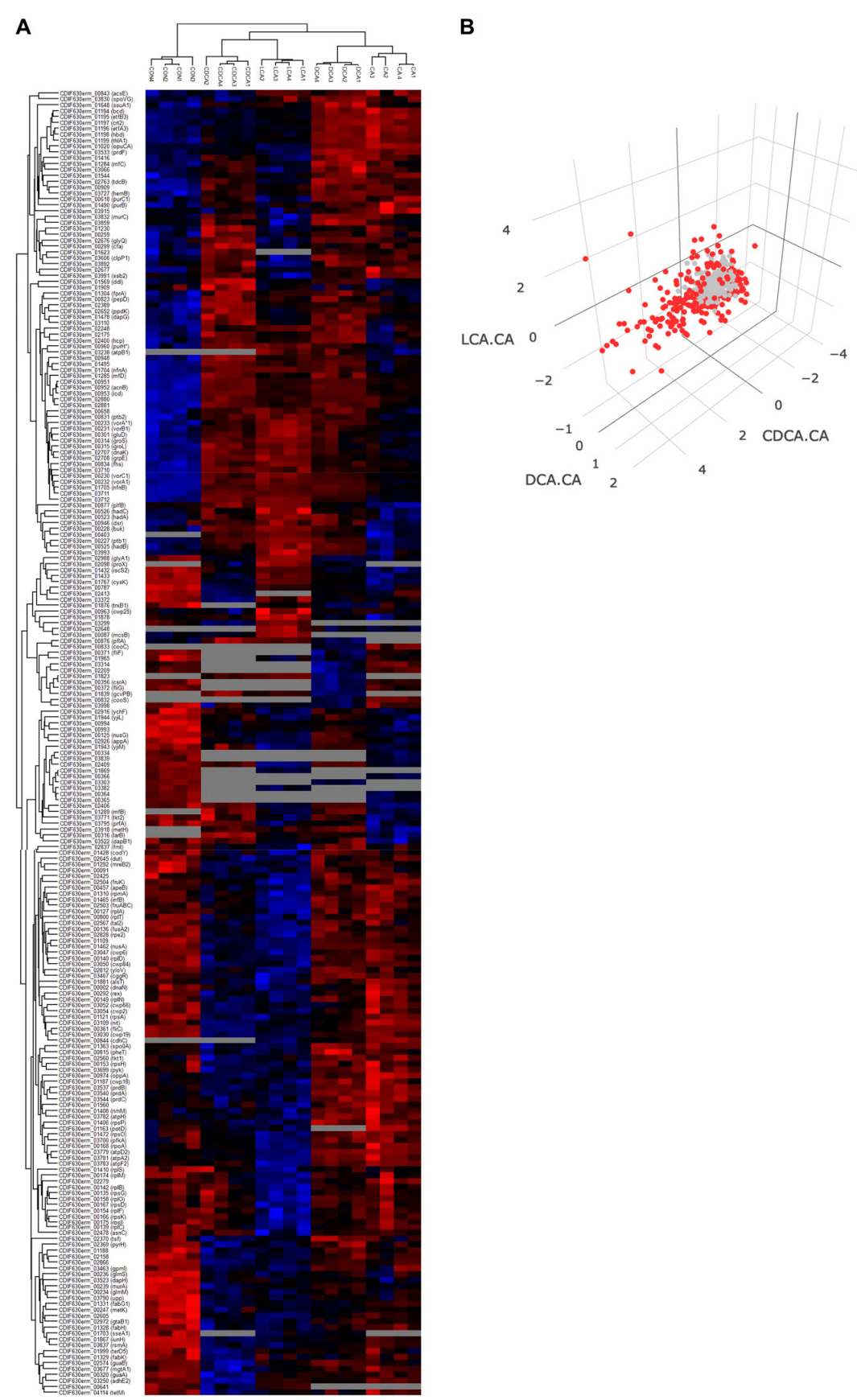

FIGURE 4 | Four replicates of control and the four bile acid samples are visualized in a heatmap (A). Only proteins quantified in all replicates of a specific condition were considered, otherwise they were grayed out. Higher and lower protein abundance is indicated in red and blue colors, respectively. For proteins that could be quantitated in all four bile acid stress conditions, ratios of protein abundance [ $\Delta \log 2(\mathrm{LCA}-\mathrm{CA}), \Delta \log 2(\mathrm{CDCA}-\mathrm{CA}), \Delta \mathrm{log} 2(\mathrm{DCA}-\mathrm{CA})]$ were calculated and visualized in a 3D-plot (B). Red data points symbolize proteins of a log2 ratio higher than 1 or below -1. In a supplementary version of Figure 4B the perspective can be rotated (Supplementary Figure S3).

of bile acids might be the result of two superimposed effects. Increasing bile acid concentrations hamper growth in general, but could also specifically interfere with flagella-driven motility. The diameter of the growth zones of $C$. difficile challenged with CA and DCA decreased linearly with increasing concentrations. Presumably, this decrease is predominantly caused by the general inhibition of growth. However, the growth zone diameter seems to drop rapidly in presence of $0.3 \mathrm{mM}$ CDCA and $0.03 \mathrm{mM}$ LCA. These concentrations have only a minor (CDCA) or no effect (LCA) on growth in liquid cultures of C. difficile and might therefore rather impair motility than growth of C. difficile. 
A

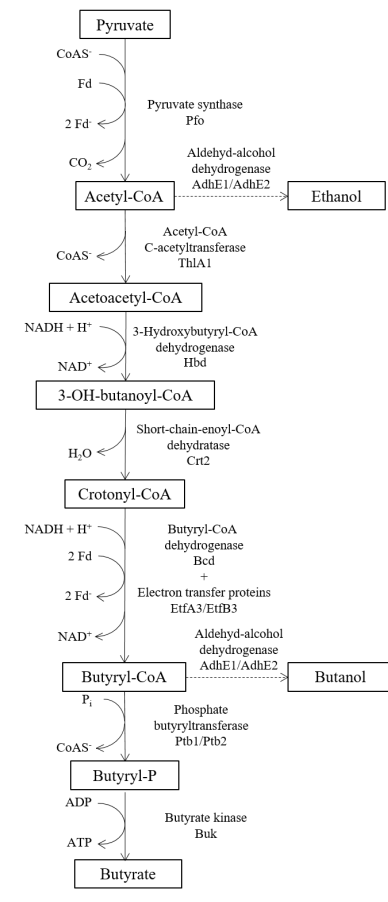

B

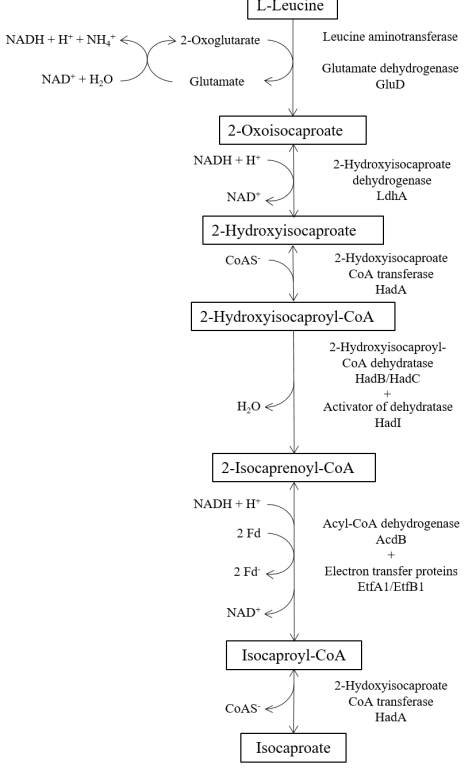

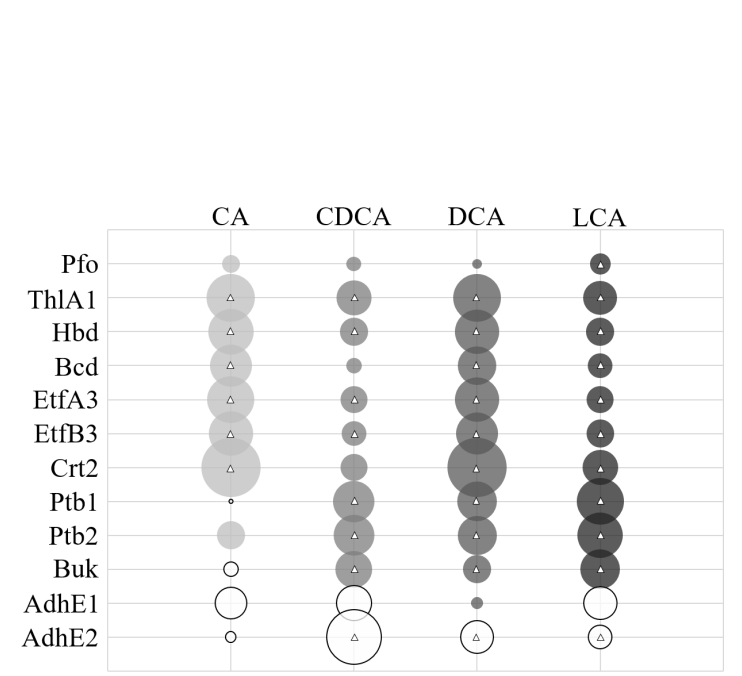

AdhE1
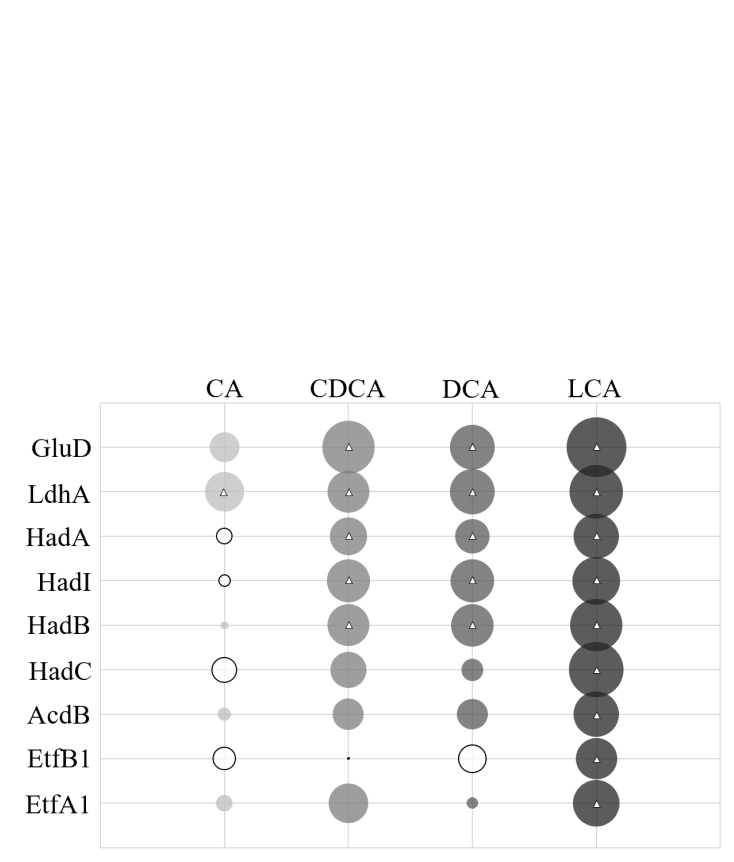

FIGURE 5 | (A) Fermentation pathway of pyruvate down to butyrate with branches to ethanol and butanol. The abundance of corresponding enzymes in relation to control conditions is symbolized in circles. Size of circles corresponds to extent of fold change (FC). Solid circles represent FCs bigger than one (bile acid/control) and open circles represent FCs less than one. Circles centered with a triangle are based on a quantitation with $p$-values $<0.05$. (B) Reductive Stickland reaction of leucine to isocaproate (adapted from Kim et al., 2005) with quantitative proteomic data of enzymes depicted in circles. Exact values of protein quantification and $p$-values are provided in Supplementary Table S4.

\section{DISCUSSION}

The amphiphilic nature of bile acids is challenging to bacterial cell integrity. At higher concentrations bile acids cause disruption of cellular membranes and subsequently cell lysis accounting for their antimicrobial activity. However, at sub-lethal concentrations bile acids might induce more specific effects on cells, e. g., a modulation of cellular signaling processes (Zhou et al., 2013). Notably, a ligand-receptor-like interaction has been described in C. difficile for taurocholic acid and the spore protein 
A

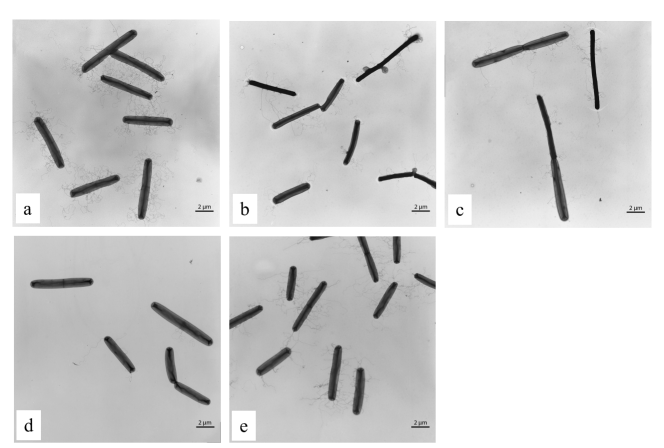

B

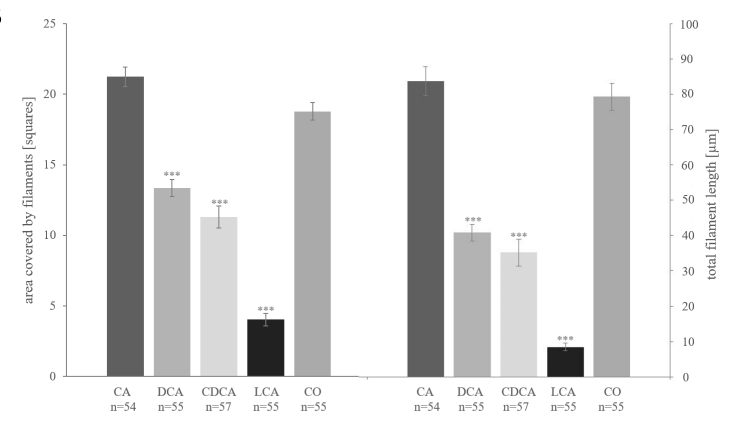

C

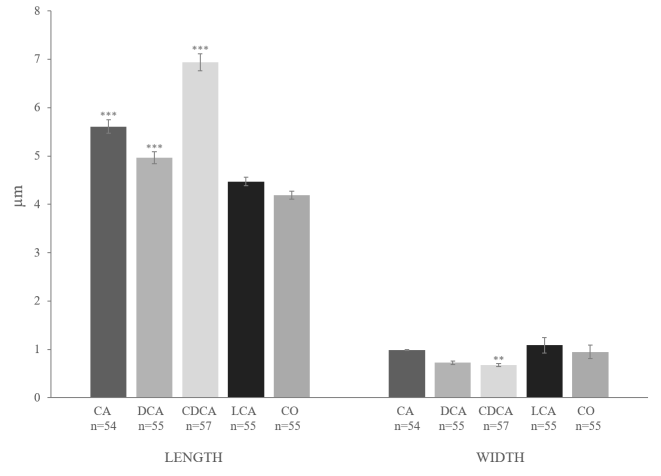

FIGURE 6 | (A) Transmission electron micrographs of $C$. difficile challenged with the four different bile acids CA (a), DCA (b), CDCA (c), LCA (d) and unstressed cells (e). (B) The covered area (left columns) and the total length (right columns) of flagella per cell were measured and are given in squares und $\mu \mathrm{m}$, respectively. (C) Average length (left columns) and width (right columns) of bacterial cells are given in $\mu \mathrm{m}$. Values significantly different in the presence of bile acid compared to non-treated cells (CO) are marked $\left({ }^{* * *} p\right.$-value $<0.001 ;{ }^{* *} p$-value $\left.<0.01\right)$

CspC, which initiates germination of the spore (Francis et al., 2013). The study presented herein aimed at the elucidation of so far unknown specific effects of selected bile acids going beyond a general detergent-like action.

As a starting point, different sub-lethal and inhibitory concentrations of the bile acids CA, DC, and CDCA were determined. While $C$. difficile tolerates high amounts of CA (6 $\mathrm{mM}$ in shock experiment), it appeared to be tenfold more sensitive to DC and CDCA. This might be due to the stronger hydrophobicity of the steroid scaffold of DCA and CDCA compared to CA featuring one additional hydroxy group which could decrease its cell-lysing capacity. It is also possible that fewer hydroxy groups facilitate the diffusion and uptake of bile acids into the cytoplasm where they can interfere with cellular processes. In this case, the tri-hydroxylated CA would mostly remain in the extracellular medium. In a follow-up study the extra- and intracellular bile acid concentrations after cell exposure will be determined by metabolic analyses of bile acids and derivatives of them. This will provide answers on how fast different bile acids enter $C$. difficile cells and if the bacterium is able to modify or even degrade them. Furthermore, an immunostaining of bile acids coupled to fluorescence- or electron microscopy will elucidate the location on the cellular surface or within the $C$. difficile cell and might disclose differences between the tested bile acids. Notably, when $C$. difficile was challenged with concentrations of bile acids higher than used in the shock experiments, cell lysis occurred. However, such concentrations could be employed for LT-stress experiments. This indicates that C. difficile is capable of adapting to bile acids by rearranging cellular structures to prevent cell lysis and even to grow in the presence of these high concentrations. In contrast to the other three bile acids, LCA could not be added to the medium in higher concentrations than $0.08 \mathrm{mM}$ due to the formation of micelles. A conjugation of LCA with a small hydrophilic compound at the carboxy-group would have allowed a testing of higher concentrations, since the CMC (critical cellular concentration) would have been raised by the conjugation. However, in this study we decided to focus on the steroid scaffold as it occurs after deconjugation of the bile acids by microbes in the intestines and therefore used the sodium salts of unconjugated bile acids. Even though the LCA-concentration used in this study only had a small growth-inhibiting effect, quantitative proteomic data clearly indicated a stress response of $C$. difficile to this bile acid.

Our data indicate that a $90 \mathrm{~min}$ bile acid shock leads to a general cellular response independent from the chemical structure of the bile acids and likely due to their overall soap-like character. However, when $C$. difficile is continuously grown in presence of bile acids, thereby mimicking the in vivo conditions in the host intestines, numerous specific cellular adaptation processes but almost no general stress response could be observed.

The amphiphilic nature of bile acids also provokes an increasing denaturation of proteins leading to an up-regulation of chaperones in the cell. One interesting finding is the differential regulation of chaperones after bile acid shock. Whilst the expression of DnaK, DnaJ, and GrpE (encoded in one operon) increases in response to any of the tested bile acids, only LCA and less pronounced CDCA result in the induction of the chaperonins GroL and GroS, ClpB, the arginine kinase McsB and the ATPase $\mathrm{ClpC}$, the latter two encoded in the operon also encoding the repressor protein CtsR. Although heat shock in $C$. difficile has been investigated yet (Ternan et al., 2012), the exact regulation of the different chaperones has not been described for $C$. difficile up to now. Notably, the respective coding operons in $C$. difficile are closely related to those of other well-studied bacteria, i.e., Bacillus subtilis (Schumann, 2016), and one would therefore predict a comparable regulation of gene expression. In B. subtilis, the dna and gro operons belong to the class I heat shock genes 

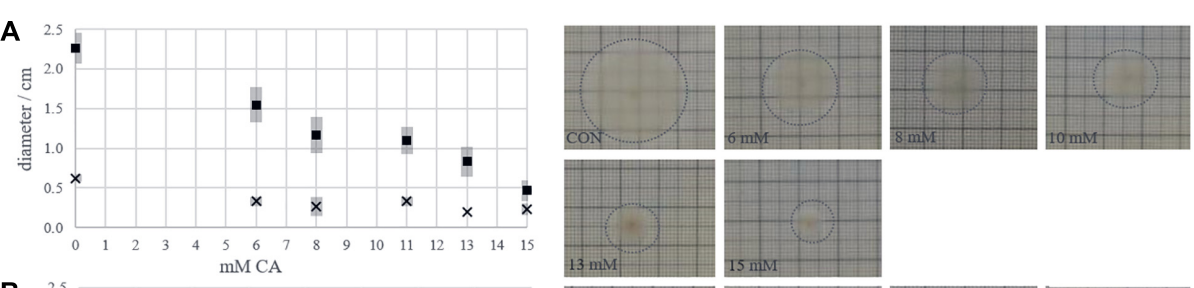

$\mathbf{B}$
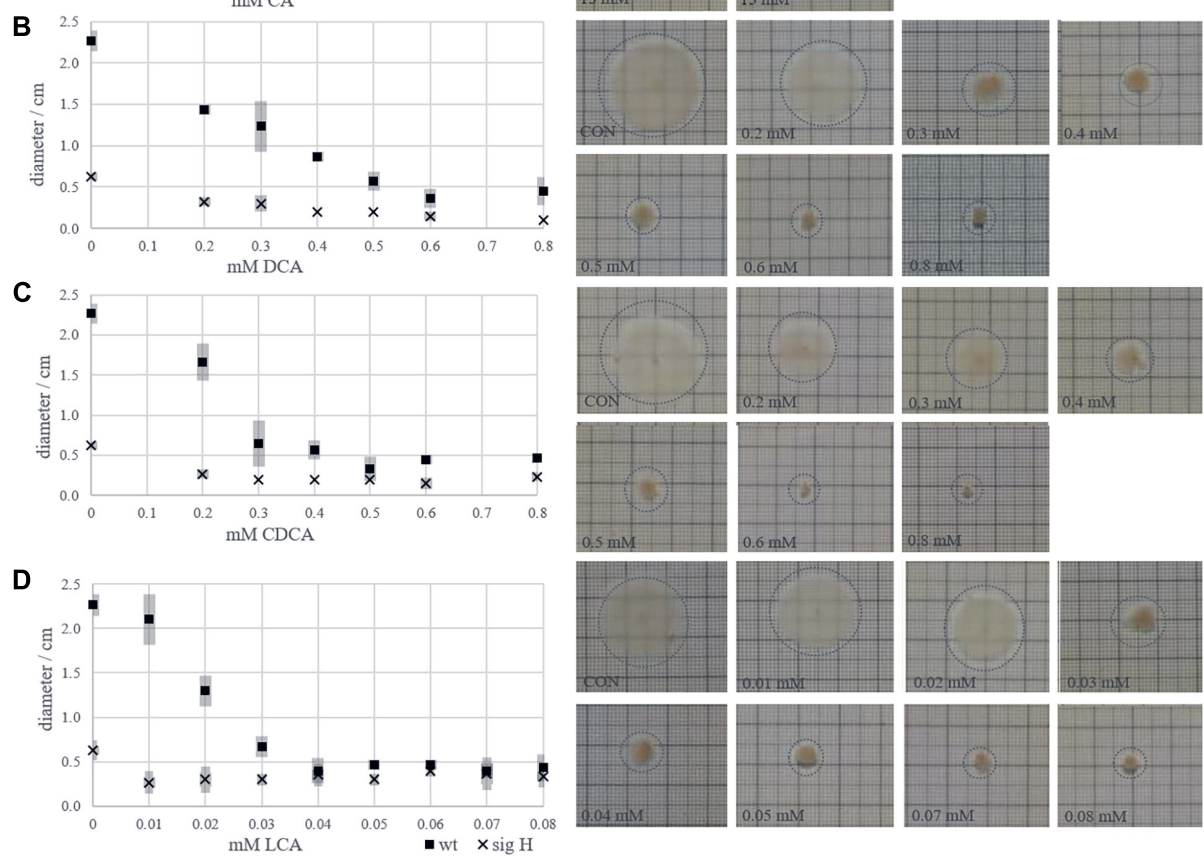

FIGURE 7 | Zones of growth of $C$. difficile inoculated in the center of soft agar plates provided with increasing concentrations of bile acids. Diameters of growth halos in dependence of bile acid concentration are given in diagrams (left) accompanied by images of exemplary agar plates (right) for CA (A), DCA (B), CDCA (C), and LCA (D).

and are controlled by the transcriptional repressor HrcA. In C. botulinum, a derepression of the dna and gro operons in a hrcA mutant is reported, but the gro locus in that mutant is further inducible by heat, pointing at another factor of regulation (Selby et al., 2011). Possibly CtsR, the transcriptional repressor controlling the class III heat shock genes, could adopt this role, since Emerson et al. (2008) observed a similar regulation of typically CtsR regulated genes and groLS in microarray analyses, and our results of bile acid shock response also support this hypothesis. The here postulated double regulation of the gro locus by the repressors HrcA and CtsR has to be proven experimentally, but implies that all tested bile acids stimulate a HrcA response, whilst only LCA and CDCA induce a CtsR response.

Not only typical stress response proteins were up-regulated in the presence of bile acids, but also the abundance of enzymes involved in central metabolic pathways was altered. In the presence of bile acids, e. g., pyruvate was rather converted to butyric acid than to alcohols such as ethanol and butanol compared to non-stress conditions. Also, a strong induction of the reductive Stickland pathway converting L-leucine to isocaproate could be observed during LT-stress with DCA, CDCA, and LCA but remarkably not with CA. Several subunits of the ATP synthase dramatically decreased in amount in the
LT-presence of CDCA and LCA, but not with CA and DCA. Likely, CDCA and LCA have a higher impact on membrane function and maintenance of the proton motive force (PMF) than DCA and CA resulting in dysregulation of enzymes that are involved in the generation of a proton gradient. The impact of bile acids on the PMF, and a possible difference depending on the sort of bile acid, will be tested by incubation of the cells with a carbocyanine-based dye whose fluorescence depends on the membrane potential. C. difficile possibly counteracts a decreased proton motive force and diminished ATP synthesis by an increased substrate level phosphorylation via the oxidation of pyruvate to acetyl-CoA for ADP phosphorylation. In this oxidation reaction ferredoxin is reduced and could be re-oxidized by the increase in leucine reduction. The higher demand of pyruvate is provided by pyruvate synthase. This enzyme contains iron sulfur clusters just as the HadBC proteins in the reductive Stickland reaction of leucine, which could be the reason for the enhanced synthesis of iron sulfur clusters during bile acids stress. This hypothesis needs to be verified in future experiments, in which the effect of surplus amounts of pyruvate and leucine on bile acids tolerance will be tested. Interestingly, also proteins of the D-proline reductase complex (PrdBAC) were determined with a strong decrease in abundance in the presence of CDCA 
and LCA. Kabisch et al. (1999) described the D-proline reductase in Clostridium sticklandii as a cytosolic selenoenzyme composed of three protein subunits. Future studies need to clarify if specific bile acids hamper formation or activity of D-proline reductase in $C$. difficile.

An important finding of this study is the differential effect bile acids have on the formation of flagella in $C$. difficile. Cells grown in the presence of CA synthesize flagella comparable to untreated cells. C. difficile stressed with the corresponding secondary bile acid DCA and the primary bile acid CDCA are characterized by fewer flagella, while cells challenged with LCA were basically completely impaired in flagella formation. A simple explanation of this observation would be that $C$. difficile is unable to build flagella because of a disturbed proton motif force or for the benefit of ATP saving. The impact of flagella on the motility, adherence and virulence for pathogenic bacteria, specifically for $C$. difficile, has been extensively discussed (Haiko and Westerlund-Wikstrom, 2013; Stevenson et al., 2015). It was shown that missing flagella, more specifically missing of the major structural component FliC, cause non-motility, but increase the adherence capability of $C$. difficile (Dingle et al., 2011) leading to an increased virulence in hamsters (Dingle et al., 2011) and mice (Barketi-Klai et al., 2014). The higher virulence could also be the consequence of a reported up-regulation of toxin synthesis in fliC mutated C. difficile cells (Aubry et al., 2012). Thus, the presence or absence of the major filament protein FliC does not only influence virulence directly by an altered motility and adhesiveness, but also has an impact on gene expression, e. g., the transcription of the pathogenicity locus encoding toxins $\mathrm{A}$ and B (Aubry et al., 2012; Barketi-Klai et al., 2014). Furthermore, due to its immunogenicity (Bruxelle et al., 2017), an absence of FliC could be advantageous during infection and help to evade the immune system. Our observation that the tested bile acids with exception of CA decrease flagella formation of $C$. difficile is thus highly relevant for the infection course. Bacteria in the upper intestinal tract face high CA concentrations, which facilitate spore germination and allow for flagellated and mobile vegetative cells. Along the intestines, the concentrations of secondary bile acids such as LCA increase leading to less flagellated bacteria that are still capable to adhere to tissue and might produce more toxins than in the small intestine or upper colon sections. Still the question remains, which regulatory mechanism leads to the absence of flagella during LCA stress but persistence of flagella even with very high levels of CA. It might simply be the stronger hydrophobicity of the steroid scaffold of LCA compared to CA that interferes with a proper assembly of flagella. However, it could also be speculated that LCA, as the most lipophilic of the tested bile acids, easily crosses the membrane and interferes intracellularly with regulatory processes controlling flagella formation, e. g., with a recently described flagellar switch (Anjuwon-Foster and Tamayo, 2017). Previously, Jain et al. investigated $C$. difficile cells mutated in the dnaK gene and found them to lack flagella (Jain et al., 2017), to be non-motile and longer than wild type cells. This phenotype very much resembles the one of some of the LT bile acid stressed cells of this study. Even though $d n a K$ expression increased in bile acid stressed cells, the higher demand of DnaK possibly results in a lack of the chaperone for its regular functions as a putative role in flagella formation (Jain et al., 2017) and cell division. Another indicator of a disturbed cell division and cell wall formation in stressed cells is the observed dysregulation of several proteins involved in peptidoglycan synthesis (MurA, MreB1 and B2) and of several cell wall-bound proteins of mostly unknown function (Cwp2/6/18/19/66/84).

The bile acids tested in this study all carry their hydroxy groups in alpha conformation as they naturally occur in the human intestines. Thus, the hydroxy groups are located on one side of the steroid skeleton resulting in a hydrophobic plane on the opposite side. Modifications of the 7-OH group of bile acids as epimerization to beta conformation (Setoguchi et al., 1984; Lepercq et al., 2004), oxidation to a keto group (Macdonald and Hutchison, 1982) or dehydroxylation (Buffie et al., 2015) have been numerously reported. Whereas an epimerization of bile acids would bring hydroxy groups onto the opposite side of the steroid scaffold and thus decrease hydrophobicity, the dehydroxylation would increase hydrophobicity and thereby the toxic character of the bile acid (Hino et al., 2001). The formation of epimers and keto groups can compete with dehydroxylation (Macdonald and Hutchison, 1982) and could prevent formation of the secondary bile acids DCA and LCA from CA and CDCA, respectively, which could have a major impact on the outcome of a $C$. difficile infection. With respect to the protective nature of the microbiota during $C$. difficile infections, it would therefore not only be beneficial to feature bacteria as $C$. scindens that can convert primary to secondary bile acids (Buffie et al., 2015), but also to avoid alternative modifications of the hydroxy group on C7 competing with the dehydroxylation reaction. C. difficile itself is not capable of a dehydroxylation on $\mathrm{C} 7$, but it is not clear if it could alternatively modify the $\mathrm{C} 7$ hydroxy group to protect from the harmful action of secondary bile acids. Altogether, multiple modifications are possible and reported on C7 of the steroid scaffold, whereas the hydroxy group on $\mathrm{C} 12$ seems to be more static and difficult to modify. Indeed, a large cluster of proteins was similar regulated in CA and DCA opposed to CDCA and LCA LT-stress, respectively. The first two bile acids feature a C12 hydroxy group, which is missing in the latter two. The presence of the C12 hydroxy group on a specific bile acid could therefore be an important determinant for the action of this bile acid.

Previous studies on the effect of bile acids on $C$. difficile mostly concentrate on the benefit of taurocholic acid for the germination of spores or generally describe a growth inhibiting effect of secondary bile acids on vegetative cells. Proteomics data obtained in this study clearly showed that there is not only a general stress response of $C$. difficile to any of the tested bile acids, but there are also specific responses depending on the kind of bile acid. Our observations do not support the idea of a simple grouping into "adverse" primary and "beneficial" secondary bile acids concerning a $C$. difficile infection, but rather indicate complex stress response networks. This study provides a global overview on the response to different bile acids and only a few selected differentially expressed proteins of this multivariate response could be discussed in more detail at this point. Our comprehensive dataset will be a starting point for future in-depth analyses in which the molecular background and the signal 
transduction pathways of the bile acid adaptation process of C. difficile will be elucidated. This knowledge will be the basis for a development of tailored analogs of bile acids which can efficiently hamper $C$. difficile growth with minimal side effects in patients.

\section{AUTHOR CONTRIBUTIONS}

SS designed the research. SD, NM, VG, and ST performed the experiments. $\mathrm{CW}, \mathrm{DZ}$, and $\mathrm{CH}$ were involved in mass spectrometric analyses. NM and RS prepared the samples for electron microscopy and recorded the pictures. DT, NM, and SS analyzed, evaluated and visualized the data. KR and SS wrote the paper.

\section{FUNDING}

This work was supported by grants of the "Ministerium für Bildung, Wissenschaft und Kultur Mecklenburg-Vorpommern“ (UG16001), the German Research Foundation [RTG 1947/1 Project C4] and the University of Greifswald. We acknowledge

\section{REFERENCES}

Anjuwon-Foster, B. R., and Tamayo, R. (2017). A genetic switch controls the production of flagella and toxins in Clostridium difficile. PLoS Genet. 13:e1006701. doi: 10.1371/journal.pgen.1006701

Aubry, A., Hussack, G., Chen, W., KuoLee, R., Twine, S. M., Fulton, K. M., et al. (2012). Modulation of toxin production by the flagellar regulon in Clostridium difficile. Infect. Immun. 80, 3521-3532. doi: 10.1128/IAI.00224-12

Barketi-Klai, A., Monot, M., Hoys, S., Lambert-Bordes, S., Kuehne, S. A., Minton, N., et al. (2014). The flagellin FliC of Clostridium difficile is responsible for pleiotropic gene regulation during in vivo infection. PLoS One 9:e96876. doi: 10.1371/journal.pone.0096876

Bartlett, J. G. (2006). Narrative review: the new epidemic of Clostridium difficileassociated enteric disease. Ann. Intern. Med. 145, 758-764.

Begley, M., Gahan, C. G. M., and Hill, C. (2005). The interaction between bacteria and bile. FEMS Microbiol. Rev. 29, 625-651. doi: 10.1016/j.femsre.2004. 09.003

Brandes, V., Schelle, I., Brinkmann, S., Schulz, F., Schwarz, J., Gerhard, R., et al. (2012). Protection from Clostridium difficile toxin B-catalysed Rac1/Cdc42 glucosylation by tauroursodeoxycholic acid-induced Rac1/Cdc42 phosphorylation. Biol. Chem. 393, 77-84. doi: 10.1515/BC-2011-198

Bruxelle, J.-F., Mizrahi, A., Hoys, S., Collignon, A., Janoir, C., and Pechine, S. (2017). Clostridium difficile flagellin FliC: evaluation as adjuvant and use in a mucosal vaccine against Clostridium difficile. PloS One 12:e187212. doi: 10.1371/journal.pone.0187212

Buffie, C. G., Bucci, V., Stein, R. R., McKenney, P. T., Ling, L., Gobourne, A., et al. (2015). Precision microbiome reconstitution restores bile acid mediated resistance to Clostridium difficile. Nature 517, 205-208. doi: 10.1038/ nature 13828

Dannheim, H., Riedel, T., Neumann-Schaal, M., Bunk, B., Schober, I., Sproer, C., et al. (2017). Manual curation and reannotation of the genomes of Clostridium difficile 630 deltaerm and C. difficile 630. J. Med. Microbiol. 66, 286-293. doi: 10.1099/jmm.0.000427

Darkoh, C., Brown, E. L., Kaplan, H. B., and DuPont, H. L. (2013). Bile salt inhibition of host cell damage by Clostridium difficile toxins. PLoS One 8:e79631. doi: 10.1371/journal.pone.0079631

Dingle, T. C., Mulvey, G. L., and Armstrong, G. D. (2011). Mutagenic analysis of the Clostridium difficile flagellar proteins, FliC and FliD, and their contribution to virulence in hamsters. Infect. Immun. 79, 4061-4067. doi: 10.1128/IAI. 05305-11

Emerson, J. E., Stabler, R. A., Wren, B. W., and Fairweather, N. F. (2008). Microarray analysis of the transcriptional responses of Clostridium difficile support for the article processing charge from the German Research Foundation (393148499) and the open access publication fund of the University of Greifswald.

\section{ACKNOWLEDGMENTS}

We are very grateful to Mareike Berges and Dieter Jahn for providing the sigH mutant of $C$. difficile $630 \Delta \mathrm{erm}$. Furthermore, we very much appreciate support by Christoph Metzendorf to work the programming language $\mathrm{R}$.

\section{SUPPLEMENTARY MATERIAL}

The Supplementary Material for this article can be found online at: https://www.frontiersin.org/articles/10.3389/fmicb. 2019.00258/full\#supplementary-material

Supplementary Figures S1, S2, S4 are provided via https://doi. org/10.6084/m9.figshare.7642763. Supplementary Figure S3 is accessed via https://doi.org/10.6084/m9.figshare.7646822.

to environmental and antibiotic stress. J. Med. Microbiol. 57, 757-764. doi: 10.1099/jmm.0.47657-0

Forster, S. C., and Lawley, T. D. (2015). Systematic discovery of probiotics. Nature Biotechnol. 33, 47-49. doi: 10.1038/nbt.3111

Francis, M. B., Allen, C. A., Shrestha, R., and Sorg, J. A. (2013). Bile acid recognition by the Clostridium difficile germinant receptor, CspC, is important for establishing infection. PLoS Pathog. 9:e1003356. doi: 10.1371/journal.ppat. 1003356

Greathouse, K. L., Harris, C. C., and Bultman, S. J. (2015). Dysfunctional families: clostridium scindens and secondary bile acids inhibit the growth of Clostridium difficile. Cell Metabol. 21, 9-10. doi: 10.1016/j.cmet.2014.12.016

Haiko, J., and Westerlund-Wikstrom, B. (2013). The role of the bacterial flagellum in adhesion and virulence. Biology 2, 1242-1267. doi: 10.3390/biology2041242

Hino, A., Morita, M., Une, M., Fujimura, K., and Kuramoto, T. (2001). Effects of deoxycholic acid and its epimers on lipid peroxidation in isolated rat hepatocytes. J. Biochem. 129, 683-689.

Hofmann, J. D., Otto, A., Berges, M., Biedendieck, R., Michel, A.-M., Becher, D., et al. (2018). Metabolic reprogramming of Clostridioides difficile during the stationary phase with the induction of toxin production. Front. Microbiol. 9:1970. doi: 10.3389/fmicb.2018.01970

Hussain, H. A., Roberts, A. P., and Mullany, P. (2005). Generation of an erythromycin-sensitive derivative of Clostridium difficile strain $630(630 \Delta \mathrm{erm})$ and demonstration that the conjugative transposon $\mathrm{Tn} 916 \Delta \mathrm{E}$ enters the genome of this strain at multiple sites. J. Med. Microbiol. 54, 137-141. doi: 10.1099/jmm.0.45790-0

Jain, S., Smyth, D., O’Hagan, B. M. G., Heap, J. T., McMullan, G., Minton, N. P., et al. (2017). Inactivation of the dnaK gene in Clostridium difficile 630 deltaerm yields a temperature-sensitive phenotype and increases biofilm-forming ability. Sci. Rep. 7:17522. doi: 10.1038/s41598-017-17583-9

Kabisch, U. C., Gräntzdörffer, A., Schierhorn, A., Rücknagel, K. P., Andreesen, J. R., and Pich, A. (1999). Identification of D-proline reductase from Clostridium sticklandii as a selenoenzyme and indications for a catalytically active pyruvoyl group derived from a cysteine residue by cleavage of a proprotein. J. Biol. Chem. 274, 8445-8454.

Karlsson, S., Burman, L. G., and Akerlund, T. (2008). Induction of toxins in Clostridium difficile is associated with dramatic changes of its metabolism. Microbiology 154, 3430-3436. doi: 10.1099/mic.0.2008/019778-0

Kim, J., Darley, D., and Buckel, W. (2005). 2-Hydroxyisocaproyl-CoA dehydratase and its activator from Clostridium difficile. FEBS J. 272, 550-561. doi: 10.1111/j. 1742-4658.2004.04498.x

Lepercq, P., Gerard, P., Beguet, F., Raibaud, P., Grill, J.-P., Relano, P., et al. (2004). Epimerization of chenodeoxycholic acid to ursodeoxycholic acid by Clostridium 
baratii isolated from human feces. FEMS Microbiol. Lett. 235, 65-72. doi: 10. 1016/j.femsle.2004.04.011

Lewis, B. B., Carter, R. A., Ling, L., Leiner, I., Taur, Y., Kamboj, M., et al. (2017). Pathogenicity locus, core genome, and accessory gene contributions to Clostridium difficile virulence. mBio 8:e00885-17. doi: 10.1128/mBio.00885-17

Lewis, B. B., Carter, R. A., and Pamer, E. G. (2016). Bile acid sensitivity and in vivo virulence of clinical Clostridium difficile isolates. Anaerobe 41, 32-36. doi: 10.1016/j.anaerobe.2016.05.010

Long, S. L., Gahan, C. G. M., and Joyce, S. A. (2017). Interactions between gut bacteria and bile in health and disease. Mol. Asp. Med. 56, 54-65. doi: 10.1016/ j.mam.2017.06.002

Macdonald, I. A., and Hutchison, D. M. (1982). Epimerization versus dehydroxylation of the 7 alpha-hydroxyl- group of primary bile acids: competitive studies with Clostridium absonum and 7 alpha-dehydroxylating bacteria (Eubacterium sp.). J. Steroid Biochem. 17, 295-303.

Martin-Verstraete, I., Peltier, J., and Dupuy, B. (2016). The Regulatory networks that control Clostridium difficile toxin synthesis. Toxins 8:E153. doi: 10.3390/ toxins 8050153

Muntel, J., Fromion, V., Goelzer, A., Maabeta, S., Mader, U., Buttner, K., et al. (2014). Comprehensive absolute quantification of the cytosolic proteome of Bacillus subtilis by data independent, parallel fragmentation in liquid chromatography/mass spectrometry (LC/MS(E)). Mol. Cell. Proteom. 13, 10081019. doi: $10.1074 / \mathrm{mcp} . \mathrm{M} 113.032631$

Neumann-Schaal, M., Metzendorf, N. G., Troitzsch, D., Nuss, A. M., Hofmann, J. D., Beckstette, M., et al. (2018). Tracking gene expression and oxidative damage of $\mathrm{O} 2$-stressed Clostridioides difficile by a multi-omics approach. Anaerobe doi: 10.1016/j.anaerobe.2018.05.018 [Epub ahead of print].

Otto, A., Maass, S., Lassek, C., Becher, D., Hecker, M., Riedel, K., et al. (2016). The protein inventory of Clostridium difficile grown in complex and minimal medium. Proteom. Clin. Appl. 10, 1068-1072. doi: 10.1002/prca.201600069

Rappsilber, J., Mann, M., and Ishihama, Y. (2007). Protocol for micropurification, enrichment, pre-fractionation and storage of peptides for proteomics using StageTips. Nat. protoc. 2, 1896-1906. doi: 10.1038/nprot. 2007.261

Ridlon, J. M., Harris, S. C., Bhowmik, S., Kang, D.-J., and Hylemon, P. B. (2016). Consequences of bile salt biotransformations by intestinal bacteria. Gut Microbes 7, 22-39. doi: 10.1080/19490976.2015.1127483

Rupnik, M., Wilcox, M. H., and Gerding, D. N. (2009). Clostridium difficile infection: new developments in epidemiology and pathogenesis. Nat. Rev. Microbiol. 7, 526-536. doi: 10.1038/nrmicro2164

Saujet, L., Monot, M., Dupuy, B., Soutourina, O., and Martin-Verstraete, I. (2011). The key sigma factor of transition phase, SigH, controls sporulation, metabolism, and virulence factor expression in Clostridium difficile. J. Bacteriol. 193, 3186-3196. doi: 10.1128/JB.00272-11

Schindelin, J., Arganda-Carreras, I., Frise, E., Kaynig, V., Longair, M., Pietzsch, T., et al. (2012). Fiji: an open-source platform for biological-image analysis. Nat. Methods 9, 676-682. doi: 10.1038/nmeth.2019

Schmidt, R., Jager, V. D., Zuhlke, D., Wolff, C., Bernhardt, J., Cankar, K., et al. (2017). Fungal volatile compounds induce production of the secondary metabolite Sodorifen in Serratia plymuthica PRI-2C. Sci. Rep. 7:862. doi: 10. 1038/s41598-017-00893-3

Schumann, W. (2016). Regulation of bacterial heat shock stimulons. Cell Stress Chaperones 21, 959-968. doi: 10.1007/s12192-016-0727-z

Seekatz, A. M., Theriot, C. M., Rao, K., Chang, Y.-M., Freeman, A. E., Kao, J. Y., et al. (2018). Restoration of short chain fatty acid and bile acid metabolism following fecal microbiota transplantation in patients with recurrent Clostridium difficile infection. Anaerobe doi: 10.1016/j.anaerobe.2018. 04.001 [Epub ahead of print].

Selby, K., Lindstrom, M., Somervuo, P., Heap, J. T., Minton, N. P., and Korkeala, H. (2011). Important role of class I heat shock genes hrcA and dnaK in the heat shock response and the response to $\mathrm{pH}$ and $\mathrm{NaCl}$ stress of group I Clostridium botulinum strain ATCC 3502. Appl. Environ. Microbiol. 77, 2823-2830. doi: 10.1128/AEM.02633-10

Setoguchi, T., Higashi, S., Tateno, S., Yahiro, K., and Katsuki, T. (1984). Epimerization of the four 3,7-dihydroxy bile acid epimers by human fecal microorganisms in anaerobic mixed cultures and in feces. J. Lipid Res. 25, 1246-1256.

Sievers, S., Dittmann, S., Jordt, T., Otto, A., Hochgrafe, F., and Riedel, K. (2018). Comprehensive redox profiling of the thiol proteome of Clostridium difficile. Mol. Cell. Proteom. 17, 1035-1046. doi: 10.1074/mcp.TIR118.000671
Sievert, C., Parmer, C., Hocking, T., Chamberlain, S., Ram, K., Corvellec, M., et al. (2016). Plotly: Create Interactive Web Graphics via 'Plotly.js' Version 4.8.0.

Sorg, J. A., and Sonenshein, A. L. (2008). Bile salts and glycine as cogerminants for Clostridium difficile spores. J. Bacteriol. 190, 2505-2512. doi: 10.1128/JB. 01765-07

Sorg, J. A., and Sonenshein, A. L. (2009). Chenodeoxycholate is an inhibitor of Clostridium difficile spore germination. J. Bacteriol. 191, 1115-1117. doi: 10. 1128/JB.01260-08

Stevenson, E., Minton, N. P., and Kuehne, S. A. (2015). The role of flagella in Clostridium difficile pathogenicity. Trends Microbiol. 23, 275-282. doi: 10.1016/ j.tim.2015.01.004

Ternan, N. G., Jain, S., Srivastava, M., and McMullan, G. (2012). Comparative transcriptional analysis of clinically relevant heat stress response in Clostridium difficile strain 630. PLoS One 7:e42410. doi: 10.1371/journal.pone.0042410

Thanissery, R., Winston, J. A., and Theriot, C. M. (2017). Inhibition of spore germination, growth, and toxin activity of clinically relevant $C$. difficile strains by gut microbiota derived secondary bile acids. Anaerobe 45, 86-100. doi: 10.1016/j.anaerobe.2017.03.004

Theriot, C. M. (2018). Beyond structure: defining the function of the gut using omic approaches for rational design of personalized therapeutics. mSystems 3:e00173-17. doi: 10.1128/mSystems.00173-17

Theriot, C. M., Bowman, A. A., and Young, V. B. (2016). Antibiotic-induced alterations of the gut microbiota alter secondary bile acid production and allow for Clostridium difficile spore germination and outgrowth in the large intestine. mSphere 1:e0045-15. doi: 10.1128/mSphere.00045-15

Theriot, C. M., Koenigsknecht, M. J., Carlson, P. E. J. R., Hatton, G. E., Nelson, A. M., Li, B., et al. (2014). Antibiotic-induced shifts in the mouse gut microbiome and metabolome increase susceptibility to Clostridium difficile infection. Nat. Commun. 5:3114. doi: 10.1038/ncomms4114

Thomas, C., Stevenson, M., and Riley, T. V. (2003). Antibiotics and hospitalacquired Clostridium difficile-associated diarrhoea: a systematic review. J. Antimicrob. Chemother. 51, 1339-1350. doi: 10.1093/jac/dkg254

Tyanova, S., Temu, T., Sinitcyn, P., Carlson, A., Hein, M. Y., Geiger, T., et al. (2016). The perseus computational platform for comprehensive analysis of (prote)omics data. Nat. Methods 13, 731-740. doi: 10.1038/nmeth.3901

Vizcaino, J. A., Csordas, A., del-Toro, N., Dianes, J. A., Griss, J., Lavidas, I., et al. (2016). 2016 update of the PRIDE database and its related tools. Nucleic Acids Res. 44:11033. doi: 10.1093/nar/gkw880

Weingarden, A. R., Chen, C., Bobr, A., Yao, D., Lu, Y., Nelson, V. M., et al. (2014). Microbiota transplantation restores normal fecal bile acid composition in recurrent Clostridium difficile infection. Am. J. physiol. Gastrointest. Liver Physiol. 306, G310-G319. doi: 10.1152/ajpgi.00282.2013

Wilson, K. H. (1983). Efficiency of various bile salt preparations for stimulation of Clostridium difficile spore germination. J. Clin. Microbiol. 18, 1017-1019.

Winston, J. A., and Theriot, C. M. (2016). Impact of microbial derived secondary bile acids on colonization resistance against Clostridium difficile in the gastrointestinal tract. Anaerobe 41, 44-50. doi: 10.1016/j.anaerobe.2016. 05.003

Yoon, S., Yu, J., McDowell, A., Kim, S. H., You, H. J., and Ko, G. (2017). Bile salt hydrolase-mediated inhibitory effect of Bacteroides ovatus on growth of Clostridium difficile. J. Microbiol. 55, 892-899. doi: 10.1007/s12275-0177340-4

Zhou, Y., Maxwell, K. N., Sezgin, E., Lu, M., Liang, H., Hancock, J. F., et al. (2013). Bile acids modulate signaling by functional perturbation of plasma membrane domains. J. Biol. Chem. 288, 35660-35670. doi: 10.1074/jbc.M113.519116

Zuhlke, D., Dorries, K., Bernhardt, J., Maass, S., Muntel, J., Liebscher, V., et al. (2016). Costs of life - Dynamics of the protein inventory of Staphylococcus aureus during anaerobiosis. Sci. Rep. 6:28172. doi: 10.1038/srep28172

Conflict of Interest Statement: The authors declare that the research was conducted in the absence of any commercial or financial relationships that could be construed as a potential conflict of interest.

Copyright (®) 2019 Sievers, Metzendorf, Dittmann, Troitzsch, Gast, Tröger, Wolff, Zühlke, Hirschfeld, Schlüter and Riedel. This is an open-access article distributed under the terms of the Creative Commons Attribution License (CC BY). The use, distribution or reproduction in other forums is permitted, provided the original author(s) and the copyright owner(s) are credited and that the original publication in this journal is cited, in accordance with accepted academic practice. No use, distribution or reproduction is permitted which does not comply with these terms. 